УДК 303.732.4+514.84+515.1+530.1

01.00.00 Физико-математические науки

ВЛИЯНИЕ РЕАКЦИИ
ДИССОЦИАЦИИРЕКОМБИНАЦИИ
МОЛЕКУЛ ВОДЫ НА ПЕРЕНОС 1:1
ЭЛЕКТРОЛИТА В МЕМБРАННЫХ
СИСТЕМАХ В ДИФФУЗИОННОМ СЛОЕ.
ЧАСТЬ 2. АСИМПТОТИЧЕСКЙ АНАЛИЗ

Коваленко Анна Владимировна к.э.н., доцент

Scopus Author ID: 55328224000

SPIN-код автора: 3693-4813

Кубанский государственный университет, Россия,350040, Краснодар, Ставропольская, 149 savanna-05@mail.ru

Уртенов Махамет Али Хусеевич д.ф.-м.н., профессор

Scopus Author ID: 6603363090

SPIN-код: 7189-0748

Кубанский государственный университет, Россия,350040, Краснодар, Ставропольская, 149, urtenovmax@mail.ru

Сеидова Наталья Михайловна

к.ф.-м.н., доцент

Scopus Author ID: 23470572500

SPIN-код автора: 8290-7153

Кубанский государственный университет,

Россия,350040, Краснодар, Ставропольская, 149 natalia.seidova@gmail.com

Письменский Александр Владимирович к.ф.-м.н., доцент

Scopus Author ID: 13004856800

SPIN-код: 9932-7747

Кубанский государственный университет,

Россия,350040, Краснодар, Ставропольская, 149, archer812@mail.ru

Данная статья посвящена асимптотическому анализу краевой задачи для системы уравнений Нернста-Планка и Пуассона для сингулярно возмущенной системы обыкновенных дифференциальных уравнений [1], зависящей еще от двух параметров. Эта краевая задача моделирует электродиффузии четырех сортов ионов одновременно в диффузионном слое в электромембранных системах с идеально селективной мембраной с учетом реакции рекомбинации двух из ионов. При этом два других иона представляют собой ионы бинарной соли. В качестве простейшего примера можно рассматривать перенос ионов натрия, хлора, водорода и гидроксила, причем ионы водорода и гидроксила рекомбинируют в диффузионном слое. Более сложным случаем является перенос
UDC $303.732 .4+514.84+515.1+530.1$

Physic and mathematics

\section{THE INFLUENCE OF REACTION OF DISSOCIATION / RECOMBINATION OF MOLECULES OF WATER ON TRANSPORTING ELECTROLYTE 1: 1 IN THE MEMBRANE SYSTEMS IN THE DIFFUSION LAYER. PART 2. ASYMPTOTIC ANALYSIS}

Kovalenko Anna Vladimirovna Cand.Econ.Sci., associate professor Scopus Author ID: 55328224000 SPIN-code: $3693-4813$

Kuban State University, Krasnodar, Russia

Urtenov Makhamet Ali Khuseevich

Dr.Sci.Phys.-Math., professor

Scopus Author ID: 6603363090

SPIN-code: 7189-0748

Kuban State University, Krasnodar, Russia

Seidova Natalia Mikhaylovna

Cand.Phys.-Math.Sci., associate professor

Scopus Author ID: 23470572500

SPIN-code: 8290-7153

Kuban State University, Krasnodar, Russia

Pismenskiy Alexander Vladimirovich

Cand.Phys.-Math.Sci., associate professor

Scopus Author ID: 13004856800

SPIN-code: 9932-7747

Kuban State University, Krasnodar, Russia

This article is devoted to the asymptotic analysis of boundary value problem for a system of equations of Nernst-Planck and Poisson for a singularly perturbed system of ordinary differential equations [1], based on two parameters. This boundary value problem simulates electrodiffusion of four kinds of ions at the same time in the diffusion layer in electro-membrane systems with perfectly selective membrane, taling into consideration the reaction of recombination of two ions. Meanwhile the other two ions represent ions of a binary salt. As a simple example, we consider the transport of ions sodium, chlorine, hydrogen and hydroxide, moreover, hydrogen and hydroxyl ions recombine in the diffusion layer. A more complex case is the transfer of the products of dissociation of the dihydrogen phosphate of sodium, namely, ions of sodium and dihydrogen phosphate, the latter dissociate 
продуктов диссоциации дигидрофосфата натрия, а именно, ионов натрия и дигидрофосфата, причем последний диссоцирует на межфазной границе в свою очередь на ионы водорода и гидрофосфата. Таким образом, в растворе одновременно могут находится три разных сорта ионов: натрия, водорода и гидрофосфата. В процессе переноса ионы водорода и гидрофосфата рекомбинируют с образованием фосфорной кислоты. В статье выявлена структура диффузионного слоя Нернста при токах, выше тока Харкаца. Показано, в диффузионном слое имеется два типа погранслоев: внутренний (реакционный) погранслой и погранслой на межфазной границе раствор/мембрана

Ключевые слова: ДИГИДРОФОСФАТ, ЭЛЕКТРОКОНВЕКЦИЯ, РЕАКЦИЯ ДИССОЦИАЦИИ, РЕАКЦИЯ РЕКОМБИНАЦИИ МОЛЕКУЛ ВОДЫ, ЭЛЕКТРОМЕМБРАННЫЕ СИСТЕМЫ, ОБЛАСТЬ ПРОСТРАНСТВЕННОГО ЗАРЯДА, КАТИОНООБМЕННАЯ МЕМБРАНА, АНИОНООБМЕННАЯ МЕМБРАНА at the interface, in turn, hydrogen ions and hydrogen phosphate. Thus, in the solution can simultaneously store three different types of ions: sodium, hydrogen, phosphate. During the transfer, hydrogen ions and ions of hydrogen phosphate recombine to produce phosphoric acid. The article has revealed the structure of the Nernst diffusion layer at currents above Harkatsa current. It is shown, that in the diffusion layer, there are two types of boundary layers: the inner (reaction) boundary layer and boundary layer at the interface solution / membrane

Doi: 10.21515/1990-4665-122-017

\section{Введение}

Данная статья посвящена асимптотическому анализу краевой задачи для сингулярно возмущенной системы обыкновенных дифференциальных уравнений [1], зависящей еще от двух параметров.

Эта краевая задача моделирует электродиффузии четырех сортов ионов одновременно в диффузионном слое в электромембранных системах (ЭМС) c идеально селективной мембраной $\mathrm{c}$ учетом реакции рекомбинации двух из ионов. При этом два других иона представляют собой ионы бинарной соли. В качестве простейшего примера можно рассматривать перенос ионов $\mathrm{Na}^{+}, \mathrm{Cl}^{-}, \mathrm{H}^{+}, \mathrm{OH}^{-}$, причем ионы водорода и гидроксила рекомбинируют в диффузионном слое. Более сложным случаем является перенос продуктов диссоциации дигидрофосфата натрия, a именно, ионов $\mathrm{Na}^{+}$и дигидрофосфата $\mathrm{H}_{2} \mathrm{PO}_{4}^{-}$, причем последний диссоцирует на межфазной границе в свою очередь на ионы водорода $\mathrm{H}^{+}$ и гидрофосфата $\mathrm{H}_{2} \mathrm{PO}_{4}^{2-}$. Таким образом, в растворе одновременно могут находится три разных сорта ионов: натрия, водорода и гидрофосфата. В http://ej.kubagro.ru/2016/08/pdf/17.pdf 
процессе переноса ионы водорода и гидрофосфата рекомбинируют с образованием фосфорной кислоты.

\section{1. Математическая постановка задачи}

Краевая задача имеет следующий безразмерный вид [1]:

$$
\begin{gathered}
\frac{d C_{1}}{d x}=C_{1} E-j_{1}, \\
\frac{d C_{2}}{d x}=-C_{2} E-j_{2}, \\
\frac{d C_{3}}{d x}=C_{3} E-j_{3}, \\
\frac{d}{d x} j_{3}=K_{3}\left(k_{w}-C_{3} C_{4}\right), \\
\frac{d C_{4}}{d x}=-C_{4} E-j_{4}, \\
\frac{d}{d x} j_{4}=K_{4}\left(k_{w}-C_{3} C_{4}\right), \\
\varepsilon \frac{d E}{d x}=C_{1}-C_{2}+C_{3}-C_{4}, \\
\left.C_{1}\right|_{x=0}=1,\left.C_{2}\right|_{x=0}=1+d,\left.C_{3}\right|_{x=0}=C_{30}=d \quad,\left.j_{3}\right|_{x=0}=j_{30},\left.j_{4}\right|_{x=0}=j_{40}=0 \\
\left.C_{1}\right|_{x=1}=C_{1 m},\left.\quad j_{3}\right|_{x=1}=j_{3 m}=0,\left.j_{4}\right|_{x=1}=j_{4 m},
\end{gathered}
$$

причем $I_{s}+I_{W}=I, \quad j_{1}=$ Cons,$\quad j_{2}=$ Cons,$\quad$ где $I_{S}=D_{1} j_{1}-D_{2} j_{2}$, $I_{W}=D_{3} j_{3}-D_{4} j_{4}$, для идеально селективной мембраны $j_{2}=0, \varepsilon>0, k_{w}>0$ малые параметры, $K>0$ большой параметр, $C_{1 m}$ равна обменной емкости катионообменной мембраны, $I_{W}=\gamma_{1} C_{1 m}^{\gamma_{2}}$.

\section{2. Алгоритм асимптотического решения}

При запредельных плотностях тока вблизи мембраны появляется область пространственного заряда (ОПЗ) $\bar{x}<x<1$, где уже условие локальной электронейтральности не выполняется и вместо него необходимо использовать уравнение Пуассона. В то же время в интервале $[0, \bar{x})$ выполняется условие электронейтральности. Предположим, что 
центр реакционного слоя $\theta$ (точка рекомбинации ионов водорода и гидроксила) расположена в области электронейтральности $(0, \bar{x})$. В окрестности этой точки появляется внутренний погранслой (узкий реакционный слой), где происходит рекомбинация молекул воды и находится решение в этом погранслое, которое сращивается с решениями в областях $(0, \theta)$ и $(\theta, \bar{x})$. Для решения краевой задачи в $(0, \bar{x})$ используется методика работы [2], которая заключается в разбиении интервала $(0, \bar{x})$ на два интервала $(0, \theta)$ и $(\theta, \bar{x})$, в которых выполняется условие равновесия. В области пространственного заряда $(\bar{x}, 1]$ краевая задача решается с использованием асимптотического метода разработанного нами в работах [6]. А именно, ОПЗ ( $\bar{x}, 1]$ разбивается на две подобласти: основную часть $(\bar{x}, €)$, где решения относительно медленно меняются и квазиравновесную часть (£,1] (погранслой), где решения экспоненциально меняются и удовлетворяют граничному условию. Вводится промежуточный слой около точки $x=\bar{x}$, который используется для сращивания решений из области электронейтральности $[0, \bar{x})$ и ОПЗ ( $\bar{x}, 1]$ •

\section{3. Упрощение вне реакционного слоя}

Используя малость параметра $k_{w}$ можно упростить уравнения для $j_{3}$ и $j_{4}$ отбрасывая его в уравнения, тогда

$$
\begin{aligned}
& \frac{d}{d x} j_{3}=-K \cdot C_{3} C_{4} / D_{3}, \\
& \frac{d}{d x} j_{4}=-K \cdot C_{3} C_{4} / D_{4}
\end{aligned}
$$

Поделим эти уравнения на $\mathrm{K}$, положим $\gamma=\frac{1}{K}<<1$, т.е. $\gamma$ малый параметр. При $\gamma \rightarrow 0$, получаем, что оба уравнения приводятся к виду:

$$
C_{3} C_{4}=0 \text {. }
$$

Отсюда следует, что вне реакционного слоя в системе существует химическое равновесие. Из уравнения (12) получаем $C_{3}=0$ или $C_{4}=0$. http://ej.kubagro.ru/2016/08/pdf/17.pdf 
Пусть $C_{3}(\theta)=C_{4}(\theta)$ и $\theta \in(0,1)$, причем с достаточно хорошей точностью можно полагать, что $C_{3}(\theta)=C_{4}(\theta)=0$, тогда с учетом краевых условий получим: $C_{4}(x)=0, x \in(0, \theta) \quad$ и $C_{3}(x)=0, x \in(\theta, 1)$.

\section{3. Решение в области электронейтральности}

\section{1. Решение в интервале $(0, \theta)$}

В интервале $(0, \theta)$ для функции $C_{4}(x) \equiv 0$ и неизвестных функций $C_{1}, C_{2}, C_{3}, E$ получаем краевую задачу, эквивалентную задаче об экзальтации предельного тока [2,3]:

$$
\begin{gathered}
\frac{d C_{3}}{d x}=C_{3} E-j_{3}, \frac{d C_{1}}{d x}=C_{1} E-j_{1}, \frac{d C_{2}}{d x}=-C_{2} E-j_{2}, C_{1}-C_{2}+C_{3}=0, \\
\left.C_{1}\right|_{x=0}=1,\left.C_{2}\right|_{x=0}=1+d,\left.C_{3}\right|_{x=0}=C_{30}=d,\left.j_{3}\right|_{x=0}=j_{30},
\end{gathered}
$$

причем $j_{3}=\frac{1}{D_{3}} I_{W}$, которая имеет аналитическое решение:

$$
\begin{gathered}
C_{1}=-\frac{1}{2 D_{1}} I_{S} x+\frac{-\frac{1}{D_{1}} I_{S}(1+d) x+2+2 d}{-J_{0} x+2+2 d}, \\
C_{2}=\frac{1}{2}\left(-J_{0} x+2+2 d\right), \\
C_{3}=-\frac{1}{2 D_{3}} I_{W} x+\frac{-\frac{1}{D_{3}} I_{W}(1+d) x+2 d(1+d)}{-J_{0} x+2+2 d}, \\
E=\frac{J_{0}}{-J_{0} x+2+2 d}, \text { где } J_{0}=\frac{1}{D_{1}} I_{S}+\frac{1}{D_{3}} I_{W}>0 .
\end{gathered}
$$

\section{2. Определение критических токов: предельного тока, токов}

\section{экзальтации и Харкаца}

Из формулы (13-16) следует, что определенное выше решение справедливо в интервале, где $-J_{0} x+2+2 d>0$. Т.е. в интервале $[0, \tilde{x})$, где $\tilde{x}=(2+2 d) / J_{0}$. Очевидно, что если положить $I_{W}=0, d=0$ и $x=1$, то из уравнения $-I_{S} / D_{1}+2=0$ получим значение безразмерного предельного тока [4] $I_{n p}=2 D_{1}$. Наличие в этой формуле безразмерного коэффициента 
диффузии катионов связана с тем, что потоки при переходе к безразмерному виду нормируются на коэффициенты диффузии [1]. Аналогично, получаем значение тока Харкаца $I_{X}=I_{n p}+\frac{D_{1}}{D_{3}} I_{e}=(2+2 d) D_{1}$, который представляет собой сумму предельного тока $I_{n p}$ и тока экзальтации [2] $I_{e x}=D_{1} I_{W} / D_{3}=2 d D_{1}$. При общих токах $I_{t o t}=I_{S}+I_{e x}$ выше тока Харкаца, т.е. $I_{t o t}>I_{X}$, полученное решение существует лишь на интервале $[0, \tilde{x})$, представляющим собой область электронейтральности, причем $\tilde{x}<1$, т.к. $\tilde{x}=I_{X} / I_{t o t}$.

Токи выше токов Харкаца для задач с учетом реакции диссоциации/рекомбинации молекул воды аналогичны запредельным токовым режимам без учета этой реакции. Поэтому в дальнейшем под запредельным режимом в моделях с учетом реакции диссоциации/рекомбинации молекул воды будем понимать именно режим с токами выше токов Харкаца. Заметим, что экзальтация предельного тока приводит к уменьшению области электронейтральности.

\section{3 Определение точки $\theta$}

Значение $\theta$ находится из условия $\left.C_{3}\right|_{x=\theta}=0$, тогда из условия электронейтральности следует $\left.C_{1}\right|_{x=\theta}=\left.C_{2}\right|_{x=\theta} \geq 0$ :

$$
\begin{gathered}
\theta=\tilde{x}-\frac{2 \sqrt{1+d\left(1-\frac{I_{s} D_{3}}{I_{w} D_{1}}\right)-d^{2} \frac{I_{w} D_{1} I_{s} D_{3}}{I_{w}{ }^{2} D_{1}{ }^{2}}}}{J_{0}}, \\
C_{1}(\theta)=C_{2}(\theta)=\sqrt{1+\left(1-\frac{I_{s} D_{3}}{I_{w} D_{1}}\right) d-\frac{I_{s} D_{3}}{I_{w} D_{1}} d^{2}} .
\end{gathered}
$$

Формулы $(17,18)$ имеют смысл при всех $d$, для которых подкоренное выражение положительно. Из формулы (17) получаем $\theta<\tilde{x}$ и точка $\theta$ лежит в области электронейтральности.

\section{4 Решение при $\theta<x<\bar{x}$}


В интервале $(\theta, \bar{x})$ для функции $C_{3}(x) \equiv 0$ и неизвестных функций $C_{1}, C_{2}, C_{4}, E$ имеем краевую задачу:

$$
\begin{gathered}
\frac{d C_{1}}{d x}=C_{1} E-j_{1}, \\
\frac{d C_{2}}{d x}=-C_{2} E-j_{2}, \\
\frac{d C_{4}}{d x}=-C_{4} E-j_{4}, \\
C_{1}-C_{2}-C_{4}=0, \\
\left.C_{1}\right|_{x=\theta+}=\left.C_{1}\right|_{x=\theta-},\left.\quad C_{2}\right|_{x=\theta+}=\left.C_{2}\right|_{x=\theta-},\left.\quad C_{4}\right|_{x=\theta}=0,
\end{gathered}
$$

причем $j_{4}=-\frac{1}{D_{4}} I_{W}$, которая имеет аналитическое решение:

$$
\begin{gathered}
E=\frac{J_{2}}{-J_{1}(x-\theta)+a}, \\
C_{1}(x)=\frac{I_{S}\left(-J_{1} x+J_{1} \theta+a\right)}{D_{1}\left(J_{1}+J_{2}\right)}-\frac{\left(-C_{10}+\frac{a I_{s}}{D_{1}\left(J_{1}+J_{2}\right)}\right) a^{\frac{J_{2}}{J_{1}}}}{\left(-J_{1} x+J_{1} \theta+a\right)^{\frac{J_{2}}{J_{1}}}} \\
C_{2}=\left(-J_{1}(x-\theta)+a\right)^{-\frac{J_{2}}{J_{1}}} a^{\frac{J_{2}}{J_{1}}} C_{2}(\theta) \\
C_{4}(x)=-\frac{I_{w}\left(-J_{1} x+J_{1} \theta+a\right)}{D_{4}\left(J_{1}-J_{2}\right)}+\frac{a I_{w}\left(-J_{1} x+J_{1} \theta+a\right)^{\frac{J_{2}}{J_{1}}}}{D_{4}\left(J_{1}-J_{2}\right) a^{\frac{J_{2}}{J_{1}}}}
\end{gathered}
$$

где $J_{1}=\frac{1}{D_{1}} I_{S}-\frac{1}{D_{4}} I_{W}, J_{2}=\frac{1}{D_{1}} I_{S}+\frac{1}{D_{4}} I_{W}, a=C_{1}(\theta)+C_{2}(\theta)=J_{0}(\tilde{x}-\theta)$.

Из (24-27) следует, что решение существует лишь на интервале $[\theta, \bar{x})$, где $\bar{x}=\theta+\frac{a}{J_{1}}=\theta+\frac{J_{0}}{J_{1}}(\tilde{x}-\theta)$. Так как, $J_{1}>0$, то $\bar{x}>\theta$.

С другой стороны, поскольку $J_{1}<J_{0}$, то $\bar{x}>\tilde{x}$, т.е. реакция рекомбинации увеличивает область электронейтральности, что объясняется уменьшением общего тока.

http://ej.kubagro.ru/2016/08/pdf/17.pdf 
Замечание 1. Если ограничится плотностями тока ниже плотности тока Харкаца, то можно полагать выполненным условие локальной электронейтральности практически во всем интервале $(0,1)$, т.е. $\bar{x}=1$ и заменять уравнение Пуассона условием локальной электронейтральности. Такая модель для 1:1 электролита для ЭМС является аналогом модели Сокирко-Харкаца для электродных систем [2].

\section{4. Нахождение напряженности электрического поля в области} пространственного заряда $\bar{x}<x<1$

В интервале $\bar{x}<x<1$ имеем систему уравнений, отличающуюся от системы (19-22) в интервале $\theta<x<\bar{x}$, только тем, что вместо условия электронейтральности (22) используется уравнение Пуассона:

$$
\varepsilon \frac{d E}{d x}=C_{1}-C_{2}-C_{4}
$$

Для системы уравнений $(19-21,28)$ ставятся краевые условия:

$$
\begin{gathered}
\left.C_{1}\right|_{x=\bar{x}+}=\left.C_{1}\right|_{x=\bar{x}-},\left.C_{2}\right|_{x=\bar{x}+}=\left.C_{2}\right|_{x=\bar{x}-},\left.\quad C_{4}\right|_{x=\bar{x}}=0, \\
\left.C_{1}\right|_{x=1}=C_{1 m} .
\end{gathered}
$$

\section{1. Вывод уравнения для $E$}

Несложно показать, что система $(19-21,28)$ имеет первый интеграл:

$$
C_{1}+C_{2}+C_{4}=\frac{1}{2} \varepsilon E^{2}-J_{1} x+\alpha,
$$

где $\alpha=C_{1}(\bar{x})+C_{2}(\bar{x})+C_{4}(\bar{x})+O(\varepsilon) . \quad \mathrm{B} \quad$ дальнейшем полагаем $\alpha \approx C_{1}(\bar{x})+C_{2}(\bar{x})+C_{4}(\bar{x})$. Используя (31) несложно получить для $E$ :

$$
\varepsilon \frac{d^{2} E}{d x^{2}}=\frac{1}{2} \varepsilon E^{3}-\left(J_{1} x-\alpha\right) E-J_{2} .
$$

\section{2 Асимптотическое решение уравнения для $E$ В $(\bar{x}, 1)$}

Основной идеей решения является разбиение интервала $(\bar{x}, 1)$ на три отрезка $\left[\bar{x}_{1}, \bar{x}_{2}\right],\left[\bar{x}_{2}, \mathfrak{E}\right]$ и $[\mathfrak{E}(\varepsilon), 1]$, причем $\left[\bar{x}_{2}, \mathfrak{E}\right]$ - основная ОПЗ, [ $\left.\bar{x}_{1}, \bar{x}_{2}\right]$ - промежуточный слой, служащий для сращивания решения в основной 
ОПЗ с решением в области электронейтральности, [E( E),1] квазиравновесная часть ОПЗ являющееся погранслоем и обеспечивающее удовлетворения краевому условию при $x=1$. В каждом отрезке, (32) решается по-своему, с использованием методики [6], а границы отрезков находятся в ходе решения:

1) $E \approx \frac{J_{2}}{\alpha-J_{1} x}$, при $x \in\left[\theta, \bar{x}_{1}\right]$, где $\bar{x}_{1}=\frac{\alpha}{J_{1}}-2 J_{1}^{-1} J_{2}^{\frac{2}{3}} \varepsilon^{\frac{1}{3}}=\bar{x}-2 J_{1}^{-1} J_{2}^{\frac{2}{3}} \varepsilon^{\frac{1}{3}}$ и совпадает с полученным выше решением в интервале $[\theta, \bar{x})$. Однако, здесь найдена точная правая граница.

2) $E \approx J_{2}^{\frac{1}{3}} \varepsilon^{-\frac{1}{3}}\left(2^{\frac{1}{3}}+\frac{1}{3} 2^{\frac{2}{3}}\left(J_{1} x-\alpha\right) J_{2}^{-\frac{2}{3}} \varepsilon^{-\frac{1}{3}}-\frac{1}{81} 2^{\frac{4}{3}}\left(J_{1} x-\alpha\right)^{3} J_{2}^{-2} \varepsilon^{-1}\right), \quad$ при $x \in\left[\bar{x}_{1}, \bar{x}_{2}\right]$, где $\bar{x}_{2}=\frac{\alpha}{J_{1}}+2 J_{1}^{-1} J_{2}^{\frac{2}{3}} \varepsilon^{\frac{1}{3}}$.

3) $E \approx \varepsilon^{-\frac{1}{2}} \sqrt{2\left(J_{1} x-\alpha\right)}$, при $x \in\left[\bar{x}_{2}, \epsilon\right]$, где $\notin(\varepsilon)=1-\gamma \sqrt{\varepsilon}|\ln \varepsilon|$.

4) $E(x)=\frac{\sqrt{2\left(J_{1}-\alpha\right)}}{\sqrt{\varepsilon}} \frac{\beta-e^{-\sqrt{2\left(J_{1}-\alpha\right)} \frac{1-x}{\sqrt{\varepsilon}}}}{\beta+e^{-\sqrt{2\left(J_{1}-\alpha\right)} \frac{1-x}{\sqrt{\varepsilon}}}}$ при [f( $\left.\left.\varepsilon\right), 1\right]$

\section{5. Нахождение концентраций в $[\bar{x}, 1]$}

Концентрации в интервале $[\theta, \bar{x})$ были найдены выше. Поскольку концентрации непрерывны в точке $\bar{x}$, а отрезок $\left[\bar{x}_{1}, \bar{x}_{2}\right]$ стягивается в эту точку при $\varepsilon \rightarrow 0+$, то концентрации по непрерывности могут быть продолжены на отрезок $\left[\bar{x}_{1}, \bar{x}_{2}\right]$. Зная $E(x, \varepsilon)$ несложно найти распределение концентраций в отрезках $\left[\bar{x}_{2}, f\right]$ и $[f(\varepsilon), 1]$, решая задачи Коши для линейных относительно $C_{1}, C_{2}, C_{4}$ уравнений (19-21). Прежде всего, отметим, что в этих отрезках $C_{2}(x, \varepsilon)=O\left(e^{-1 / \sqrt{\varepsilon}}\right)$, т.е. $C_{2}(x, \varepsilon) \approx 0$, при 
$x \in\left[\bar{x}_{2}, 1\right]$. Асимптотическое решение начального порядка для остальных концентраций в отрезке $\left[\bar{x}_{2}, \mathfrak{f}\right]$ имеют вид:

$$
\begin{aligned}
& C_{1}(x, \varepsilon)=I_{S} \sqrt{\varepsilon} / D_{1} \sqrt{2\left(J_{1} x-a\right)}, \text { при } x \in\left[\bar{x}_{2}, \epsilon\right], \\
& C_{4}(x, \varepsilon)=I_{W} \sqrt{\varepsilon} / D_{4} \sqrt{2\left(J_{1} x-a\right)} \text {, при } x \in\left[\bar{x}_{2}, £\right] \text {. }
\end{aligned}
$$

Асимптотическое решение начального порядка для концентрации $C_{1}$

B $[f(\varepsilon), 1]: C_{1}(x, \varepsilon)=C_{1 m} \cdot(\beta+1)^{2} e^{-\sqrt{2\left(J_{1}-\alpha\right)} \frac{1-x}{\sqrt{\varepsilon}}} /\left(\beta+e^{-\sqrt{2\left(J_{1}-\alpha\right)} \frac{1-x}{\sqrt{\varepsilon}}}\right)^{2}$.

Для концентраций $C_{2}$ и $C_{4}$ нет погранслоя из-за отсутствия граничного условия для $C_{4}$ при $x=1$, поэтому решения при $x \in\left[\bar{x}_{2}, \hat{\epsilon}\right]$ непрерывно продолжаются и на отрезок [ $£(\varepsilon), 1]$.

6. Решение в области реакционного слоя около $x=\theta$

\section{1. Общие свойства решений в реакционном слое}

Концентрации по построению решения являются непрерывными в точке $x=\theta$, а поскольку в $[0, \theta)$ и $(\theta, \bar{x}]$ выполняется условие локальной электронейтральности, то по непрерывности и в точке $x=\theta$ будет выполняется условие локальной электронейтральности. Таким образом, в начальном приближении по концентрациям не будет погранслоя в точке $x=\theta$. Иначе обстоит дело с потоками $j_{3}, j_{4}$ и напряженностью электрического поля $E$. Так как, в $(0, \theta): C_{4} \equiv 0, j_{4} \equiv 0, j_{3}=I_{W} / D_{3}$, a в $(\theta, \bar{x}): \quad C_{3} \equiv 0, \quad j_{3} \equiv 0, \quad j_{4}=-I_{W} / D_{4}$, то в реакционном слое происходит резкое изменение потоков $j_{3}, j_{4}$. Таким образом, реакционный слой представляет собой внутренний погранслой по потокам.

Рассмотрим теперь напряженность электрического поля. Так как в $[0, \theta)$ имеем $E(x)=\frac{J_{0}}{-J_{0} x+2+2 d}$, а в $(\theta, \bar{x}]$ имеем $E(x)=\frac{J_{2}}{-J_{1}(x-\theta)+a}$, то непосредственные вычисления показывают, что

$$
E(\theta-0)=\frac{I_{s} D_{4}+I_{w} D_{1}}{I_{s} D_{3}+I_{w} D_{1}} E(\theta+0) .
$$


Поскольку $D_{3} \neq D_{4}$, то $E(\theta-0) \neq E(\theta+0)$, и поэтому в реакционном слое, в начальном приближении также имеется внутренний погранслой по напряженности электрического поля $E$, т.е. будет наблюдаться резкое изменение $E$ между значениями $E(\theta-0)$ и $E(\theta+0)$.

\section{2. Построения напряженности $E$ в реакционном слое}

Для построения напряженности $E$ в реакционном слое, воспользуемся уравнениями для $E$ в интервалах $[0, \theta)$ и $(\theta, \bar{x}]$ и используя методику [6] найдем решения слева и справа от точки $x=\theta$ : $E_{\theta-}(\xi)=A_{1} e^{-\sqrt{\alpha-J_{0} \theta} \xi}+A_{2} e^{+\sqrt{\alpha-J_{0} \theta} \xi}+J_{0} / \alpha-J_{0} \theta$,
$E_{\theta+}(\varsigma)=A_{3} e^{-\sqrt{a-J_{1} \theta} \varsigma}+A_{4} e^{+\sqrt{a-J_{1} \theta} \varsigma}+J_{2} / a-J_{1} \theta$, где $\xi=(\theta-x) \sqrt{\varepsilon}, \quad \varsigma=(x-\theta) / \sqrt{\varepsilon}$.

Из условия сращивания этих решений с решениями вне реакционного слоя получаем при $\xi \rightarrow+\infty$ и $\varsigma \rightarrow+\infty$, определяем, $A_{2}=0$ и $A_{4}=0$, а также границы внутреннего погранслоя по $E$ : $[\theta-\sqrt{\varepsilon}|\ln \varepsilon|, \theta+\sqrt{\varepsilon}|\ln \varepsilon|]$. Из условий непрерывности $E$ и $E^{\prime}$ в точке $x=\theta$ :

$$
A_{1}+J_{0} /\left(\alpha-J_{0} \theta\right)=A_{3}+J_{2} /\left(a-J_{1} \theta\right), \quad A_{1} \sqrt{\alpha-J_{0} \theta}=A_{3} \sqrt{a-J_{1} \theta} .
$$

определяем $A_{1}$ и $A_{3}$. Таким образом:

$$
\begin{gathered}
E(x, \varepsilon)=A_{1} e^{-\sqrt{\alpha-J_{0} \theta} \frac{\theta-x}{\sqrt{\varepsilon}}}+J_{0} /\left(\alpha-J_{0} \theta\right), \quad x \in[\theta-\sqrt{\varepsilon}|\ln \varepsilon|, \theta], \\
E(x, \varepsilon)=A_{3} e^{-\sqrt{a-J_{1} \theta} \frac{x-\theta}{\sqrt{\varepsilon}}}+J_{2}\left(a-J_{1} \theta\right), x \in[\theta, \theta+\sqrt{\varepsilon}|\ln \varepsilon|] .
\end{gathered}
$$

\section{3. Построения потоков в реакционном слое $j_{3}, j_{4}$}

Для построения потоков $j_{3}, j_{4}$ в реакционном слое, воспользуемся уравнениями для потоков в общем виде. Поскольку $D_{3} j_{3}-D_{4} j_{4}=I_{w}$, то достаточно рассмотреть одно из них. Кроме того, здесь нельзя упрощать уравнения отбрасывая $k_{w}$, но тогда нужно считать, что $C_{3}(\theta)=C_{4}(\theta)=\sqrt{k_{w}}$. 
Рассмотрим уравнение (4). Дифференцируя его еще раз по $x$, с учетом уравнений для $C_{3}$ и $C_{4}$ получим $\gamma \frac{d^{2}}{d x^{2}} j_{3}=\frac{C_{4} D_{4}+C_{3} D_{3}}{D_{3} D_{4}} j_{3}-\frac{C_{3} I_{w}}{D_{3} D_{4}}$.

При $x \in(0, \theta): C_{4} \equiv 0$, поэтому имеем уравнение:

$$
\gamma \frac{d^{2}}{d x^{2}} j_{3}=\frac{C_{3}}{D_{4}} j_{3}-\frac{C_{3} I_{w}}{D_{3} D_{4}} .
$$

Вблизи точки $x=\theta-$ имеем $C_{3}(\theta-)=\sqrt{k_{w}}$, поэтому поделим обе части уравнения на $\sqrt{k_{w}}$, тогда уравнение примет вид: $\frac{\gamma}{\sqrt{k_{w}}} \frac{d^{2}}{d x^{2}} j_{3}=\frac{C_{3}}{\sqrt{k_{w}} D_{4}} j_{3}-\frac{C_{3} I_{w}}{\sqrt{k_{w}} D_{3} D_{4}} \quad$ или $\mu \frac{d^{2}}{d x^{2}} j_{3}=\frac{C_{3}}{\sqrt{k_{w}} D_{4}} j_{3}-\frac{C_{3} I_{w}}{\sqrt{k_{w}} D_{3} D_{4}}$, где $\mu=\frac{\gamma}{\sqrt{k_{w}}}=\frac{1}{\Lambda} \ll<1$ малый параметр.

Сделаем замену $\xi=(\theta-x) / \sqrt{\mu}, j_{3}(x, \mu)=j_{3}(\xi, \mu)$, тогда получим

$$
\frac{d^{2}}{d \xi^{2}} j_{3}=\frac{C_{3}(\theta-\xi \sqrt{\mu})}{\sqrt{k_{w}} D_{4}} j_{3}-\frac{C_{3}(\theta-\xi \sqrt{\mu}) I_{w}}{\sqrt{k_{w}} D_{3} D_{4}},
$$

переходя к пределу при $\mu \rightarrow 0+$, при каждом фиксированном $\xi$, получаем

$$
\begin{aligned}
& \frac{d^{2}}{d \xi^{2}} j_{3}=\frac{C_{3}(\theta)}{\sqrt{k_{w}} D_{4}} j_{3}-\frac{C_{3}(\theta) I_{w}}{\sqrt{k_{w}} D_{3} D_{4}}, \text { или с учетом } C_{3}(\theta)=\sqrt{k_{w}} \text { получим: } \\
& \frac{d^{2}}{d \xi^{2}} j_{3}=\frac{1}{D_{4}} j_{3}-\frac{I_{w}}{D_{3} D_{4}}, \text { которое имеет общее решение: } \\
& j_{3, \theta-}=A_{1} e^{-\frac{1}{\sqrt{D_{4}}} \xi}+A_{2} e^{\frac{1}{\sqrt{D_{4}}} \xi}+\frac{I_{w}}{D_{3}} .
\end{aligned}
$$

Из условия сращивания $j_{3, \theta-} \mathrm{c} j_{3}=I_{w} / D_{3}$ при $\xi \rightarrow+\infty$, получаем $A_{2}=0$. Таким образом: $j_{3, \theta-}=A_{1} e^{-\frac{1}{\sqrt{D_{4}}} \frac{\theta-x}{\sqrt{\mu}}}+I_{w} / D_{3}$, при $x \in[\theta-\sqrt{\mu}|\ln \mu|, \theta]$. 
При $x \in(\theta, \bar{x}): \quad C_{3} \equiv 0$, поэтому имеем уравнение: $\gamma \frac{d^{2}}{d x^{2}} j_{3}=\frac{C_{4}}{D_{3}} j_{3}$, решая которое по такой же схеме получаем: $j_{3, \theta+}=A_{3} e^{-\frac{1}{\sqrt{D_{4}}}} \frac{x-\theta}{\sqrt{\mu}}$, при $x \in[\theta, \theta+\sqrt{\mu}|\ln \mu|]$.Постоянные $A_{1}=-\frac{\sqrt{D_{4}}}{\left(\sqrt{D_{3}}+\sqrt{D_{4}}\right)} \cdot \frac{I_{w}}{D_{3}}, A_{3}=\frac{\sqrt{D_{3}}}{\left(\sqrt{D_{3}}+\sqrt{D_{4}}\right)} \cdot \frac{I_{w}}{D_{3}}$ находятся из условия непрерывности $j_{3}(x)$ и $j_{3}^{\prime}(x)$ в точке $x=\theta$.

Замечание 2. Толщина реакционного слоя примерно равна $2 \sqrt{\mu}=\frac{2}{\sqrt{K \sqrt{k_{w}}}}=\frac{2}{\sqrt{\Lambda}}=\frac{2}{10^{2} \sqrt{10}} \approx \frac{1}{150}$, т.е. реакционный слой занимает одну стопятидесятую часть отрезка [0,1] и поэтому можно считать его точкой.

Заключение. В статье предложен новый метод асимптотического анализа краевых задач в диффузионном слое для системы уравнений Нернста-Планка и Пуассона с учетом реакции рекомбинации ионов.

Выявлена структура диффузионного слоя Нернста при токах, выше тока Харкаца. Показано, что в диффузионном слое имеется два типа погранслоев: внутренний (реакционный) погранслой и погранслой на межфазной границе раствор/мембрана. Реакционный слой, представляет собой внутренний погранслой по потоком ионов водорода и гидроксила. В реакционном слое, также имеется внутренний погранслой по напряженности электрического поля $E$, т.е. будет наблюдаться резкое изменение («всплеск») $E$. Размеры погранслоев по потокам и напряженности в реакционном слое разные. Погранслой на межфазной границе раствор/мембрана возникает по напряженности электрического поля и по концентрации противоионов (ионов натрия).

Исследование выполнено при финансовой поддержке РФФИ в рамках научных проектов № 16-08-00128 A "Теоретическое $u$ экспериментальное исследование гравитаџионной конвекиии в мембранных системах с учетом реакиии диссоциации/рекомбинации молекул воды", № 16-48-230856 p_a "Теоретическое и экспериментальное 
исследование влияния тепловых эффектов на гравитаџионную конвекцию в мембранных системах с амфолитами".

\section{Библиографический список}

1. Коваленко А.В. Влияние реакции диссоциации/рекомбинации молекул воды на перенос 1:1 электролита в мембранных системах в диффузионном слое. Часть 1 . Математическая модель / Коваленко А.В., Уртенов М. Али Х., Сеидова Н.М., Письменский А.В. // Политематический сетевой электронный научный журнал Кубанского государственного аграрного университета (Научный журнал КубГАУ) [Электронный ресурс]. - Краснодар: КубГАУ, 2016. - №07(121). - Режим доступа: http://ej.kubagro.ru/2016/07/pdf/122.pdf, 0,813 у.п.л. - IDA [article ID]: 1211607122. http://dx.doi.org/10.21515/1990-4665-121-122

2. Сокирко А.В., Харкаца Ю.И. К теории эффекта экзальтации миграционного тока в кислых средах//«Электрохимия», 1989, т.XXV, вып.2, стр.232-239

3. Заболоцкий В.И., Шельдешов Н.В., Гнусин Н.П. Диссоциация молекул воды в системах с ионообменными мембранами// Успехи химии. 1988, т. 57, № 8, с. 1403-1414.

4. Заболоцкий В.И., Никоненко В.В. Перенос ионов в мембранах. М.: Наука, 1996. 392 c.

5. Заболоцкий В.И., Никоненко В.В., Корженко Н.М., Сеидов Р.Р., Уртенов М.Х. Влияние гетеролитической диссоциации воды на массоперенос ионов соли в электромембранной системе при нарушении электронейтральности в области диффузионного слоя. // Электрохимия. 2002. Т.38, №8. С.912-921

6. Коваленко А.В., Уртенов М.Х. Краевые задачи для системы электродиффузионных уравнений. Часть 1. -LAP Lambert Academic Publishing GmbH \& Co. KG, Germany, Saarbrucken, 2011.-281

\section{References}

1. Kovalenko A.V. Vlijanie reakcii dissociacii/rekombinacii molekul vody na perenos 1:1 jelektrolita $\mathrm{v}$ membrannyh sistemah $\mathrm{v}$ diffuzionnom sloe. Chast' 1. Matematicheskaja model' / Kovalenko A.V., Urtenov M. Ali H., Seidova N.M., Pis'menskij A.V. // Politematicheskij setevoj jelektronnyj nauchnyj zhurnal Kubanskogo gosudarstvennogo agrarnogo universiteta (Nauchnyj zhurnal KubGAU) [Jelektronnyj resurs]. - Krasnodar: KubGAU, 2016. - №07(121). - Rezhim dostupa: http://ej.kubagro.ru/2016/07/pdf/122.pdf, 0,813 u.p.l. - IDA [article ID]: 1211607122. http://dx.doi.org/10.21515/1990-4665-121-122

2. Sokirko A.V., Harkaca Ju.I. K teorii jeffekta jekzal'tacii migracionnogo toka v kislyh sredah//«Jelektrohimija», 1989, t.XXV, vyp.2, str.232-239

3. Zabolockij V.I., Shel'deshov N.V., Gnusin N.P. Dissociacija molekul vody v sistemah s ionoobmennymi membranami// Uspehi himii. 1988, t. 57, № 8, s. 1403-1414.

4. Zabolockij V.I., Nikonenko V.V. Perenos ionov v membranah. M.:Nauka,1996.392 s.

5. Zabolockij V.I., Nikonenko V.V., Korzhenko N.M., Seidov R.R., Urtenov M.H. Vlijanie geteroliticheskoj dissociacii vody na massoperenos ionov soli v jelektro-membrannoj sisteme pri narushenii jelektronejtral'nosti v oblasti diffuzionnogo sloja. // Jelektrohimija. 2002. T.38, №8. S.912-921

6. Kovalenko A.V., Urtenov M.H. Kraevye zadachi dlja sistemy jelektrodiffuzionnyh uravnenij. Chast' 1. -LAP Lambert Academic Publishing GmbH \& Co. KG, Germany, Saarbrucken, 2011.-281 